\title{
It is a differentiation game: STAT5 in a new role
}

\author{
Barbara Maurer ${ }^{1,2}$, Matthias Farlik ${ }^{3}$ and Veronika Sex| ${ }^{\star, 4}$ \\ Cell Death and Differentiation (2017) 24, 953-954; doi:10.1038/cdd.2017.71; published online 12 May 2017
}

Katerndahl, Heltemes-Harris and colleagues present a novel mechanism by which signal transducer and activator of transcription 5B (STAT5B) contributes to the pathogenesis of $B$ cell diseases: by blocking B cell differentiation. They postulate that STAT5 interferes with IKAROS and NF- $k$ B by competing for common sites including super-enhancers of genes. Experimental evidence is provided by using a Stat5b-CA mouse model, where STAT5B is rendered constitutively active in the lymphoid compartment. ${ }^{1}$ This comment discusses their findings and the potential impact on basic and clinical research.

The development and differentiation of B cells from common lymphoid progenitors is coupled to signaling pathways enabling immunoglobulin gene rearrangement and shutdown of alternate cell fates. Pre-B cells, expressing the pre-B cell receptor $(B C R)$, further differentiate to immune-competent $B$ cells - a pre-process that is orchestrated by a complex interplay of transcription factors such as PAX5, IKAROS, EBF1, E2A or PU.1. ${ }^{2}$ Deregulation of these factors is associated with transformation and development of $B$ cell acute lymphoblastic leukemia (B-ALL).

STAT5 is overexpressed and hyper-activated in a variety of hematopoietic malignancies. ${ }^{3-6}$ The key role of STAT5 in the pathogenesis and maintenance of B-ALL was substantiated in mouse studies. ${ }^{7-9}$ Despite this evidence, the mechanism underlying STAT5's prominent role in B-ALL remained only partially understood. It is well established that STAT5 regulates important factors mediating survival and proliferation, including $c-M y c, B c / 2$ and $D$ type cyclins. However, rescue experiments with these factors failed to restore STAT5's capacity to ensure viability and proliferation. ${ }^{3}$

A disease-driving role for STAT5B was recently substantiated by the identification of somatic STAT5B mutations in patients suffering primarily from T and NK cell leukemias and lymphomas. $^{10}$ Although the Stat5b-CA mouse model employed by Farrar and colleagues does not represent any of these mutations, it clearly mimics the pre-leukemic stage that is characterized by expansion of mature T and NK cells and an increase in pro-B cells. A switch to a B-ALL only occurs with very low penetrance of $2-10 \% .{ }^{11}$ The Stat $5 b-C A$ mouse model has been successfully used in the past to identify a critical role of Blnk, ${ }^{8}$ Ebf1 or Pax $5^{9}$ for STAT5B-driven B-ALL development. The significance of PAX5 is underscored by the frequent deletion in patients and by the fact that the differentiation block is restored by Pax 5 re-expression. ${ }^{12}$ Further cooperative STAT5 partners for B-ALL induction include IKAROS, SOS1, KDM2A, JAK1 or CDKN2A as recently shown using sleeping beauty transposons. ${ }^{13}$

In their current study, the authors extend these findings and unveil that Stat5b-CA functions in B cell transformation by blocking differentiation. The spontaneously evolving pre-B leukemias in Stat5b-CA mice show deregulation of critical components of the pre-BCR signaling pathway. This prompted the authors to undertake several mouse crosses. As expected, combination of Stat5b-CA with the loss of pre-BCR signaling molecules (BLNK, BTK and PKC $\beta$ ) dramatically increased

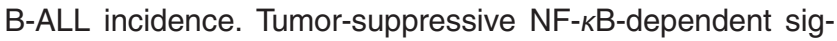
naling was hampered. In line, a sub-fraction of NF- $\kappa$ B target genes was downregulated in Stat5b-CA tumors. STAT5 ChIPSeq uncovered overlapping binding sites of STAT5 and NF- $k$ B. Upon IL-7 stimulation, STAT5 binding to the immunoglobulin $\mathrm{K}$ chain in Stat5b-CA x Blnk ${ }^{+/-}$leukemia cells was increased while NF- $k B$ binding decreased. This led them to conclude that STAT5B and NF- $k$ B compete for binding at certain common target genes (Figure 1). Integration of STAT5 ChIP-Seq with microarray data of Stat5b-CA $\times B n k^{+/-}$leukemia cells substantiated their concept.

Next, they focused on IKAROS - an NF- $k$ B-regulated key factor in lymphoid development, which is frequently lost in B-ALL. Analogous to NF- $k \mathrm{~B}$, IKAROS- and STAT5-binding motifs were found in close proximity in B-ALL cells. The comparison of IKAROS ChIP-Seq data obtained in pre-B cells with STAT5 ChIP-Seq revealed about 450 genes with a STAT5IKAROS-binding overlap. Of note, 180 of them were regulated reciprocally in Stat5b-CA-driven ALL cells. Again, a competition in binding was observed between STAT5 and IKAROS IL-7 stimulation enhanced STAT5 and reduced IKAROS binding at the Cish promoter (Figure 1).

Transcription factors do not act alone but depend on chromatin structures and act in a complex interplay with other transcriptional regulators. As such, STAT5 interacts with p300 that acetylates histone $\mathrm{H} 3$ at lysine 27 (H3K27ac) thereby promoting transcription. Vice versa, IKAROS recruits the deacetylating complex NURD (Figure 1). The authors provide a first glimpse in these chromatin alterations, which require further in-depth analysis in future studies.

\footnotetext{
${ }^{1}$ Ludwig Boltzmann Institute for Cancer Research, Vienna, Austria; ${ }^{2}$ Department for Biomedical Sciences, Institute of Animal Breeding and Genetics, University of Veterinary Medicine, Vienna, Austria; ${ }^{3}$ CeMM Research Center for Molecular Medicine of the Austrian Academy of Sciences, Vienna, Austria and ${ }^{4}$ Department for Biomedical Sciences, Institute of Pharmacology and Toxicology, University of Veterinary Medicine, Vienna, Austria

*Corresponding author: V Sexl, Institute of Pharmacology and Toxicology, University of Veterinary Medicine, Veterinaerplatz 1, Vienna 1210, Austria. Tel: +43 12 5077 2910; Fax: +43 125077 2990; E-mail: veronika.sexl@vetmeduni.ac.at
} 


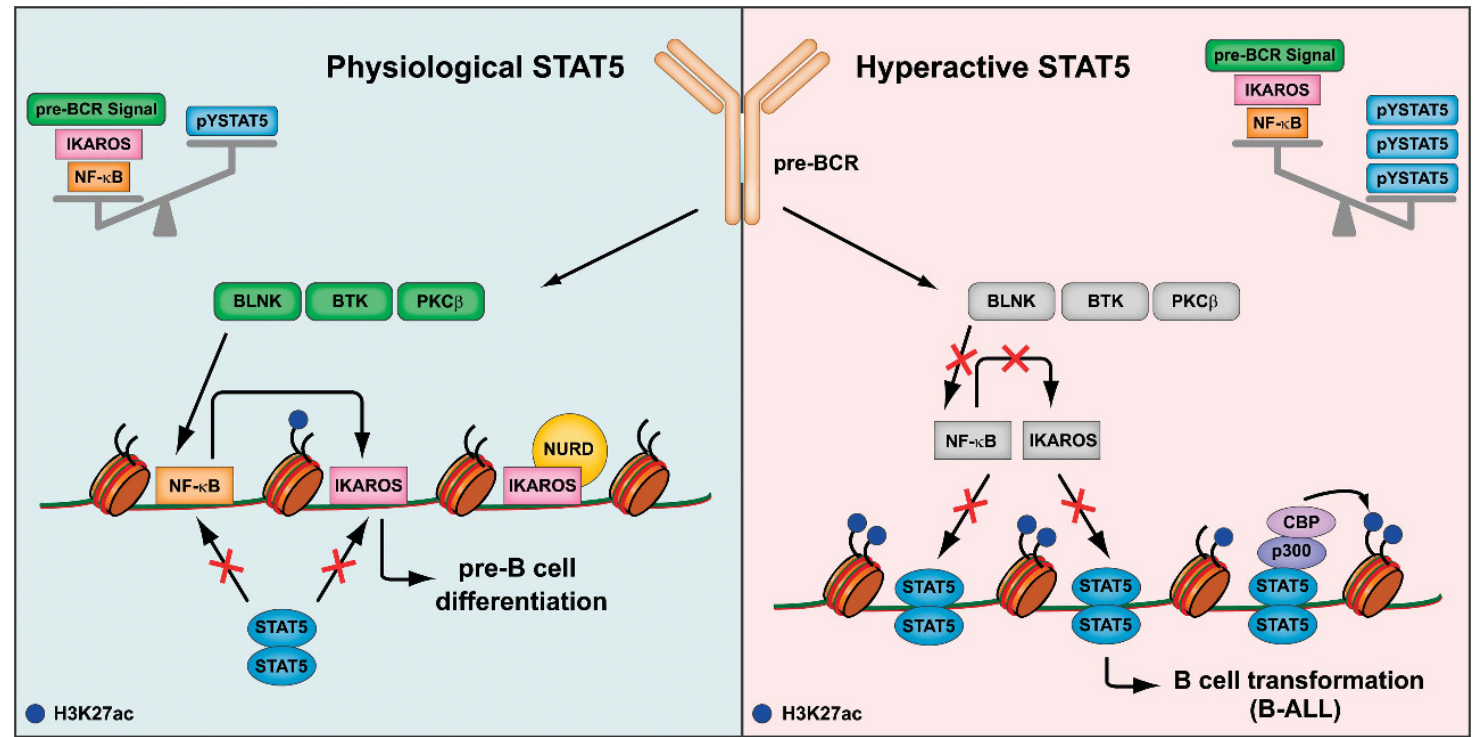

Figure 1 A new role of STAT5B in B cell differentiation and B-ALL. Left: Physiological STAT5B level. The pre-BCR signaling cascade induces NF- $\kappa B$ binding. NF- $\kappa B$ upregulates IKAROS, which recruits the deacetylating NURD complex. Correct expansion and differentiation of B cells. Right: Hyperactivation of STAT5B. Loss of pre-BCR signaling components: reduced binding of NF-KB or IKAROS, perturbation of the tightly controlled balance with STAT5 at common binding sites. Increased binding of STAT5 causes enhanced H3K27 acetylation (via p300/CBP recruitment) and leads to transformation (B-ALL)

IKAROS is part of a network of transcriptional regulators that determine lymphoid cell fate. Regulators of the epigenetic landscape of pre-B cells include the $\mathrm{B}$ cell master factors PAX5, EBF1, PU.1 and IRF4 that are present at B cell-specific super-enhancers and safeguard differentiation. ${ }^{2}$ Superenhancers, formerly known as locus control regions, are genomic regions comprising many enhancers bound by various transcription factors that regulate the expression of genes by 3D chromosomal interactions over long stretches of DNA. Focusing on the Myc locus and its super-enhancer region, the authors show that STAT5 and IKAROS are both bound. IL-7 stimulation enhanced STAT5 binding at the superenhancer but had no effect on IKAROS binding. Hence, the authors assigned opposing $M y c$ super-enhancer regulation by STAT5 and IKAROS by different histone modifications and binding to non-overlapping sites. At other locus control regions STAT5 seems to act in concert with B lineage transcription factors; STAT5 bound up to $70 \%$ of B cell super-enhancer regions in the Stat5b-CA $\times B^{\prime} k^{+/-}$setting as confirmed by ChIP-qPCR on Myc, Bcl2/1 and Igll1 superenhancer loci. It remains to be determined how STAT5 supports transcription and differentiation on some super-enhancers and - at the same time - blocks differentiation by binding to other sites.

Leukemia patients might be classified in different risk groups according to their STAT5, IKAROS and NF- $k$ B levels. Indeed, Farrar and colleagues confirmed high active STAT5 and low IKAROS levels can be used to stratify B-ALL patients - they have a worse prognosis justifying an aggressive therapeutic scheme. A next step will be to evaluate how patients carrying somatic STAT5B mutations may benefit from these findings.

In summary, this study illustrates that perturbation of two opposing transcriptional programs has fundamental consequences for the development and transformation of B cells. It is tempting to speculate that these opposing mechanisms also occur in other lineages and contribute to disease development. It remains to be elucidated whether competing binding mechanisms affect only the enforced expression of STAT5B or extend to its close homolog STAT5A. To date, STAT5B gain-offunction mutations are predominately found in T and NK cell leukemias/lymphomas. ${ }^{10,14,15}$ At present, it is entirely unclear why mutations in patients are more frequent in STAT5B than STAT5A. Normal T and NK cell development requires STAT5: in the absence of STAT5, CD8 ${ }^{+}$T cells and mature NK cells are not efficiently produced. The idea of STAT5 being a key part of a transcriptional network governing expansion and differentiation of B cells is exciting and will help to increase our understanding for the pathophysiology of B-ALL.

\section{Conflict of Interest}

The authors declare no conflict of interest.

Acknowledgements. We thank Andrea Hoelbl-Kovacic (University of Veterinary Medicine Vienna, Austria) for critical reading of the comment. BM, MF and VS are supported by the network grant FWF-SFB F061 (F6105, F6102 and F6107) from the Austrian Science Fund.

\footnotetext{
1. Katerndahl CDS et al. Nat Immunol 2017 (advance online publication).

2. Hu Y et al. Genes Dev 2016; 30: 1971-1990.

3. Hoelbl A et al. EMBO Mol Med 2010; 2: 98-110.

4. Warsch W et al. Blood 2011; 117: 3409-3420.

5. Walz C et al. Blood 2012; 119: 3550-3560.

6. Roberts KG et al. N Engl J Med 2014; 371: 1005-1015.

7. Joliot V et al. Oncogene 2006; 25: 4573-4584.

8. Nakayama J et al. Blood 2009; 113: 1483-1492.

9. Heltemes-Harris LM et al. J Exp Med 2011; 208: 1135-1149.

10. Küçük $C$ et al. Nat Commun 2015; 6: 1-12.

11. Burchill MA et al. J Immunol 2003; 171: 5853-5864.

12. Liu GJ et al. Genes Dev 2014; 28: 1337-1350.

13. Heltemes-Harris LM et al. Oncogene 2016; 35: 3454-3464.

14. Hoelbl A et al. Blood 2006; 107: 4898-4906.

15. Gotthardt D et al. Cancer Discov 2016; 6: 414-429.
} 